\title{
Mobile Ontology: Towards a Standardized Semantic Model for the Mobile Domain
}

\author{
Claudia Villalonga ${ }^{1}$, Martin Strohbach ${ }^{1}$, Niels Snoeck ${ }^{2}$, Michael Sutterer ${ }^{3}$, \\ Mariano Belaunde ${ }^{4}$, Ernö Kovacs ${ }^{1}$, Anna V. Zhdanova ${ }^{5}$, Laurent Walter Goix ${ }^{6}$, \\ and Olaf Droegehorn ${ }^{3}$ \\ ${ }^{1}$ NEC Europe Ltd., Germany \\ lastname@netlab.nec.de \\ ${ }^{2}$ Telematica Instituut, The Netherlands \\ Niels.Snoeck@telin.nl \\ ${ }^{3}$ University of Kassel, Germany \\ lastname@uni-kassel.de \\ ${ }^{4}$ France Telecom, France \\ mariano.belaunde@orange-ftgroup.com \\ ${ }^{5} \mathrm{ftw}$ - Telecommunications Research Center Vienna, Austria \\ zhdanova@ftw.at \\ ${ }^{6}$ Telecom Italia, Italy \\ laurentwalter.goix@telecomitalia.it
}

\begin{abstract}
Ontologies will be crucial for the future development of Next Generation Service Delivery Platforms. While various projects have defined ontologies for the mobile domain, there is yet little agreement on a common semantic model. One reason is the intrinsically hard problem of finding, using, mapping and evolving already existing ontologies. In this paper we present the Mobile Ontology, an effort within the IST project SPICE to converge towards a standardized ontology. Our approach is based on a minimal core ontology that defines common concepts for sub-ontologies of relevant domains and that is easily extensible towards existing and future ontologies. It is our intention to make this ontology available to other projects and collaboratively work on standardized ontology for the mobile domain.
\end{abstract}

Keywords: Ontology, SPICE platform, mobile communications, Service Delivery Platform, Next Generation Networks.

\section{Introduction}

Next Generation Networks (NGN) will rely heavily on a Service Delivery Platform (SDP) as an important component for fast and easy service creation and delivery. NGN SDPs will be increasingly complex and enable operators to open their infrastructures and interact with a large number of external systems of different organizations, ultimately creating a marketplace for value-added service enablers and services. Collaboration on such a scale requires a precise definition of shared interfaces. Past experiences have shown that pure interface definitions or reference points are not sufficient to capture the semantics needed to (automatically or semi-automatically) 
understand the offered interfaces as well as the data communicated over these interfaces [1].

In distributed software architectures and especially service oriented architectures (SOA) the problem of exchanging information between distributed software components is increasingly addressed by using ontologies to convey the semantics of interfaces and exchanged data.

In this paper we identify the need for a standardized ontology that describes semantic models of the domains relevant for scalable NGN service delivery platforms. In particular, we provide an overview of the Mobile Ontology ${ }^{l}$ and highlight the advantages gained by defining a semantic model.

Ontologies have been used extensively and some of them have even been standardized. However, these ontologies often poorly relate to each other and only apply to restricted domains, i.e. they do not sufficiently cover concepts necessary for the mobile domain. For instance, the SOUPA ontology [2] has not taken into consideration the users' needs and the fact that the ontology poorly supports mobile services and applications. Therefore, a scalable ontology infrastructure with pluggable subontologies where anyone can add on their schemata would be needed. Other projects of similar kind include GUMO [3] and SUMO [4].

The Mobile Ontology is an effort within the IST project SPICE towards resolving these issues and offering other interested parties to reuse its concepts as applicable.

The Mobile Ontology has been designed based on three major criteria:

1. Practical relevance,

2. Incorporation of existing standards and ontologies, and

3. Extensibility towards future relevant domains

Special care has been taken to include only those domains and concepts that are relevant for future service delivery platforms. In the remainder of this paper we consequently motivate the need of the most important domains and concepts and relate the ontology to concrete use cases and sub-systems of the SPICE architecture. Examples of these domains are service description, user profiles, content, context management, description of resources in the vicinity of the user and policy management and trust. The design of the Mobile Ontology is further governed by focusing on the reuse of existing ontologies (e.g. time and location) or in cases in which those are not available, on developing ontologies stemming from non-semantic data models (e.g. presence). Finally, an important design criterion has been to define an extensible ontology that allows the integration of other ontologies for future relevant domains.

\section{Overview of the Mobile Ontology}

In this section we provide an overview of the Mobile Ontology which comprises a core ontology and several sub-ontologies (Fig. 1). The extensibility of the Mobile Ontology and its structure in sub-ontologies for different domains creates the need of having some central point ontology that defines the main concepts of the mobile domain and from which the other sub-ontologies inherit. Due to the large scale of the

\footnotetext{
${ }^{1}$ Mobile Ontology. Available at http://ontology.ist-spice.org/
} 


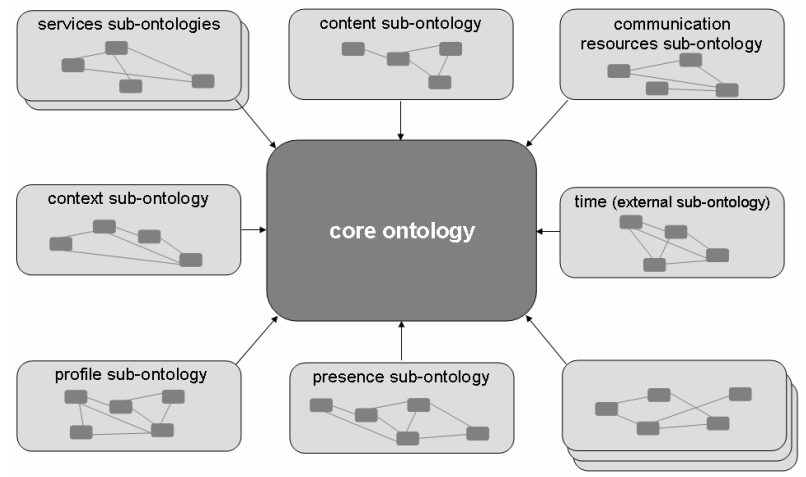

Fig. 1. Mobile Ontology

mobile domain covered by the Mobile Ontology, it makes sense to classify its concepts in a hierarchy of classes in order to make the ontology more readable and intuitive. The Mobile Ontology core plays these roles, it is the linking point between subontologies that apply to different domains and it defines a structure and the main mobile communication concepts from which all the sub-ontologies inherit.

The core ontology contains the most relevant concepts for a modern service delivery platform and that are used by all sub-ontologies. Examples include the user of the platform, people, devices and services.

In detail the SPICE ontology distinguishes between identifiable SpiceEntities and non-identifiable AbstractConcepts. While a number of concepts such as User must be globally and uniquely addressable, other individuals only exist as complex values of an entity, e.g. the address of a user. SpiceEntities are further classified into spatial and virtual entities. SpatialEntities do have a location and physical dimensions while VirtualEntities do not. Examples of SpatialEntities are the Person and Place concepts, and Service and User are sub classes of VirtualEntity. The distinction between identifiable entities and abstract concepts has been adopted from the IST MAGNET Beyond project [5].

All top-level sub-ontology classes must be subclasses of exactly one of these two concepts or its subclasses. This means that when we include existing ontologies we have to sub-class its top-level classes accordingly. From our experience this is comparable little extra effort and has the benefit of avoiding the unnecessary generation of globally unique identifiers. As the core ontology defines only a very limited amount of concepts (currently 13 classes and 7 properties) we expect that there is only very little remodeling effort needed when including external ontologies.

Sub-ontologies that have so far been defined in the SPICE project are:

- Services: specifies the description of the service, some extra information for service composition, service roaming and its context information for service execution.

- Profile: describes a user profile structure for service and situation-related profiles

- Content: description of (multimedia) content and meta information 
- Presence: reachability information of a user

- Context: describes context information

- Communication Resources: describes communication resources in the vicinity of a user that can be used to provide services to the user

We consider these ontologies to be essential for the mobile domain, but clearly expect that further sub-ontologies, e.g. for policy management, will be defined to cover the mobile domain more comprehensively.

\section{Description of the Mobile Ontology}

In this section we describe important sub-ontologies that are currently actively developed in SPICE. Due to space restrictions we are not describing other ontologies currently under development such as the content ontology. Other ontologies, e.g. access control and identity and trust and management are part of future work.

\subsection{Services}

The provisioning of advanced, high-level services is the goal of Service Delivery Platforms. The Services sub-ontology covers different domains like service description, service context, service composition and service roaming. And therefore, the Service sub-ontology can be divided into sub-ontologies which can be plugged into the core independently.

Due to the big amount of services and their complexity, it is necessary to have a semantic description of the service (e.g. OWL-S ${ }^{2}$ or WSDL-S ${ }^{3}$ ) not only to understand its interfaces but also include semantics in the discovery process. In order to provide intelligent services that offer the best experience to the user, the service has to be able to adapt to the system internal context during service execution, so the context of the service is also modeled in the service sub-ontology. Likewise semantic service composition languages allow advanced reasoning and optimization of dynamically composed services. Finally, a service roaming ontology captures concepts such as user or operator level agreements in order to enable reasoning about joining foreign network domains as well as information about the currently used domain.

\subsection{Profile}

Various services require user information, in particular situation-dependent user preferences, to customize their services to the user's wishes and expectation. It would be a hassle if each service requested and managed the required user information on its own and if the user would have to provide the required personal data to each single service. This would not only be a burden to the user during the instantiation phase but also later for the management and modification of existing user data.

Hence, it would be very beneficial from the user's point of view if these services could share the same set of user data. This can be achieved through the use of

\footnotetext{
${ }^{2}$ OWL-S. Available at http://www.daml.org/services/owl-s/1.1/

${ }^{3}$ WSDL-S. Available at http://www.w3.org/Submission/WSDL-S/
} 
common semantics. Therefore, the profile sub-ontology aims at providing a common user profile structure throughout the service platform that enables the inclusion of multiple service-related sub-profiles. On the other hand, it yet allows extending the profile structure with different user attribute vocabularies such as $\mathrm{FOAF}^{4}$. Mappings between the user attribute vocabularies can then be used for the translation between specific user instances. Such mappings can also be applied in the roaming situation, in which a user profile or parts thereof shall be transferred from the home service platform to a visited service platform.

In addition, the profile sub-ontology provides a means for describing situationdependent user preferences or sub-profiles. This will be supported in such a way, that on the one hand the user can easily specify conditions describing a specific situation and that on the other hand these conditions are machine processible so that the activation of situation-dependent user preferences can be carried out automatically by the platform instead of manually by the user.

\subsection{Presence}

Presence is used to convey information about how to contact and communicate with a person. Typical examples are instant messenger applications that display the availability of communication buddies. IETF has defined presence more accurately and standardized an exchange format, a data model and an extensible set of presence information (RFC 3863, 4479 and 4480). Examples of such presence information include the activity of a user, information about its communication devices, the type of place a person is in, information about the time zone, etc. The Open Mobile Alliance (OMA) has endorsed the IETF standards defining a 'Simple Presence Enabler'. In the IP Multimedia Subsystem (IMS) the presence standards of IETF and OMA play an important role for offering presence services to mobile users. As a consequence of this extensive standardization, presence is often used to exchange information about users and their devices.

The presence sub-ontology maps the presence standards to the semantic model defined by the Mobile Ontology. This way we reuse existing standards for modeling reachability information rather than redefine our own models. NGN service delivery platforms can benefit from the presence sub-ontology in two ways: (1) Explicit semantic modeling of presence information facilitates the process of making ontology instances available to presence-based clients. Sub-components of the SDP are not forced to implement the presence standards, but can rely on the semantic model and use dedicated translation gateways. (2) Vice versa service enablers can easily reuse standardized presence information as they only need to comply with the semantic model. Presence-based clients are not forced to provide ontology instances, but can leave translation tasks to a dedicated gateway.

\subsection{Context}

In order for intelligent services to adapt to and anticipate on the end-user's situation, gathering and interpretation of contextual information about the user and his environment is required. Such information can be obtained from various sources, ranging

${ }^{4}$ FOAF Vocabulary Specification, July 2005. Available at http://xmlns.com/foaf/0.1/ 
from sensors on the user's terminal to knowledge bases on the platform. The gathered context information can be combined and interpreted with reasoning techniques to obtain a higher level view of the user's situation. Sensors and reasoning components that facilitate this process are typically distributed throughout the platform, where each component is specialized in gathering or inferring a particular type of contextual information, utilizing the results of other components in the process. To discover and exchange information between context gathering and reasoning components, a common data format is needed that defines both the syntax and semantics of the contextual information at hand. Representing this information in an ontology ensures that all components have a common understanding of the contextual information that is exchanged. Besides specifying the types of information that can be exchanged, the ontology provides generic mechanisms for expressing information about the quality of the contextual information (QoC). This allows reasoning components and intelligent services to take additional characteristics of real-world context information into account, such as the accuracy of a sensor measurement or the probability of correctness of an inference.

\subsection{Communication Resources}

The Distributed Communication Sphere (DCS) of a user refers to the collection of communication resources in the vicinity of a user that can potentially be used to provide high-level services to that user [6]. Such resources include devices (public or owned by the user, e.g. terminals, sensors, displays, etc.), networks (e.g. networks allowing the user to access the platform and local networks like Bluetooth networks which are not directly visible from the platform), services offered by the local devices (e.g. multimodal services or Bluetooth services) and other users.

The SDP relies on semantic information about those resources in order to ensure the best possible user experience. For instance, consider a user watching a movie on his handset. If he approaches a larger display and the platform is aware of this output resource, the movie could be transferred to this display depending on its current usage, the environment and the media that should be displayed. Detailed information about the display and its current usage as well as the media is necessary in order to make decisions about how to render multimedia streams. Using an ontology to describe resources greatly facilitates the decision making process of how to render content requested by the user.

\section{SPICE Architecture}

The SPICE project targets a service platform that supports convergence between IMSbased services as well as Internet-based technologies. It defines several key areas for convergence such as federated identity management, user profiles, session management, knowledge handling, roaming support, service lifecycle management, service exposure, service brokering, and a unified service execution environment [7].

The SPICE architecture follows the design principle of horizontal layered systems that separates functionality and promotes the re-use of existing components. Fig. 2 presents an overview of the SPICE architecture for the terminal and the network. 


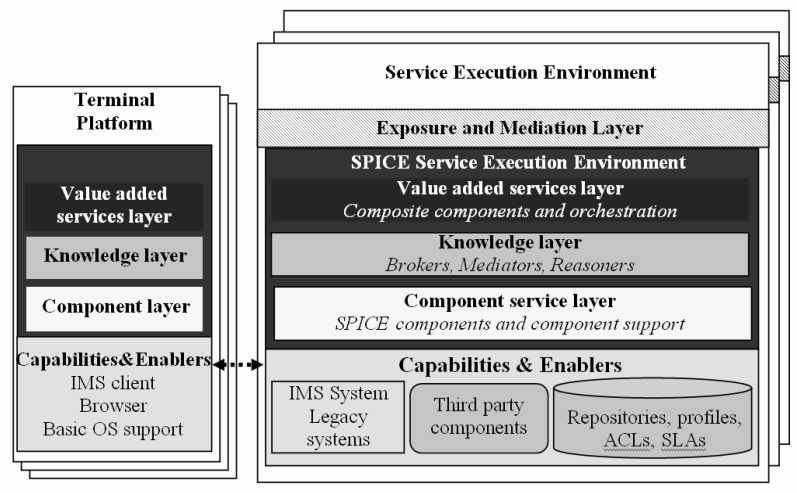

Fig. 2. Overview of the SPICE architecture

The capabilities and enablers layer is responsible for providing the various support functions that are external for the SPICE platform. They realize functions such as profile storage and management, and basic signaling and session control. Functionalities from this layer are exposed in the SPICE platform through a corresponding SPICE component (Resource Adaptor).

The component service layer provides facilities for component-based development and deployment. This layer includes services such as the service broker, various managers, controllers, and the resource adapters. The SPICE component model and methodology support cross-system service deployment and provisioning. In order to invoke component services seamlessly, component metadata and interface semantics must be developed and published in a heterogeneous environment. This approach combines the architecture of SOA (Service-Oriented Architecture) and semantic technologies.

The knowledge layer supports the discovery, delivery, and transformation of information, such as context and presence variables. This layer introduces several fundamental building blocks for intelligent components, e.g., knowledge brokers, reasoners and recommenders.

The VAS layer facilitates the creation of compound components and services from the basic SPICE components (service mash-ups). A compound component is needed, for example, for personalized ticket booking or for finding nearby restaurants that have events that match the consumer's interests. These interactions require that the services and components of several different stakeholders need to be contacted. Orchestration is needed to coordinate the information flow between these entities. Runtime meta-data and context-based discovery of components is used to find suitable components for composition.

In addition, the platform features a virtual Mediation and Exposure layer that is used to interface external platforms and resources. This virtual layer is exposing components implemented in the Component, Knowledge or VAS layer and mediates access from and to the SPICE platform. 


\section{The Mobile Ontology in SPICE}

In this section we present examples of how the mobile ontology is used in SPICE. For reasons of space restrictions we limit ourselves to two examples. The first example is a semantic model for the SPICE service composition language (SPATEL) and the second example explains how the knowledge layer in SPICE relies on ontologies.

\subsection{Service Composition}

The SPICE project has defined a high-level language for designing composite services, i.e. services that are built from existing service components. The language called SPATEL [8] - stands for SPice AdvancedLanguage for TELecommunication services - has an abstract syntax and various concrete syntaxes (like a textual format and a UML-like graphical form). This language has two main usage scenarios: it can be used by a service designer to define "manually" the logic of a service composition or it can be used by an automatic composition engine to produce the logic of the composition based on semantic annotations attached to the model elements making the service description.

The ontologies, and the Mobile Ontology in particular, relate to this SPATEL language in two ways: First, the concepts of the SPATEL language, originally defined by means of a MOF metamodel ${ }^{5}$, have been reformulated as an OWL ontology, which constitutes a sub-ontology for the Mobile Ontology. The kind of concepts found in this ontology are typical service design notions like ServiceInterface, ServiceOperation, ServiceEvent, ServiceContract, ServiceUsage, Dialogs and so on. Having these notions in the form of an ontology allows the different tools exploiting SPATEL descriptions to take advantage of ontology techniques to reason on SPATEL definitions.

Second, SPATEL service description have the possibility to embed various kinds of semantic and QoS annotations to enable scenarios where automatic discovery and selection of services are needed. These annotations are in general provided in the form of explicit links to the elements of an ontology. For instance the "goal" annotation for a service GsmLocator will typically refer to the Location element within the Mobile Ontology. Notice this approach is similar to the approach used in WSDL-S specifications.

Since there are various ways for annotating semantically services, in SPATEL a flexible mechanism based on semantic-patterns allows to accommodate to different strategies. For instance a service description may declare the usage of the well-know GIOPE pattern (Goal/Input/Output/PreCondition/Effect) to indicate that the service specification contains these set of annotations for each service operation. This declaration allows an automatic composition engine to be aware of the kind of semantic data that is available so that it can appropriately exploit it to achieve its work.

\subsection{Knowledge Management}

The SPICE knowledge layer provides a range of intelligent service enablers that provide knowledge such as presence, context, recommendations, service descriptions,

\footnotetext{
${ }^{5}$ Meta Object Facility (MOF) 2.0 Core Specification (formal/06-01-01), OMG 2006. Available at http://www.omg.org
} 
user profiles, etc. - in short every kind of knowledge that is modeled with the Mobile Ontology. One of the major challenges for knowledge management is to provide scalable access to a large number of distributed knowledge sources. In SPICE this is achieved by a knowledge discovery and exchange enabler that allows knowledge sinks to access knowledge provided by knowledge sources. Using the Context subontology enables sinks to exactly specify the information they are interested in and facilitates automated reasoning on the semantics of the data. As an example we briefly describe how the profile, the presence and the DCS sub-ontologies are used in SPICE. Other examples of intelligent service enablers include various reasoners, learners, predictors, recommenders and push service enablers [9] also make use of the Context sub-ontology.

The profile sub-ontology is used by Profile Management Enabler that manages the service and situation-specific user information of various platform services. In addition, this enabler is capable of triggering the change of situation-dependent user preferences to services based on the change of the user's situation. Furthermore, all services that query and receive user profile information require the profile subontology for correct interpretation and handling of data. Finally, the profile ontology is also needed from components involved in the roaming scenario, in particular the Roaming Manager of home and visited service platforms, in order to map user information to the required semantics used within the different service platforms.

In SPICE we have developed a presence gateway that bi-directionally maps presence information between intelligent service enablers in the knowledge layer and presence-based clients [10]. The presence gateway subscribes to presence sources and knowledge sources in the SPICE knowledge layer and transforms incoming notifications between the respective XML documents. The gateway offers a control interface to determine the information being mapped. The presence sub-ontology is therefore an important element for optimally supporting IMS and other presence-based applications. This way even legacy devices and applications that are not SPICE clients can interoperate with the platform to a certain degree.

In SPICE we are developing a resource discovery system that detects and provides information about the resources in the vicinity of the user. Resources are discovered locally by a user terminal and the RDS acts as a knowledge source providing instances of the DCS ontology to the knowledge layer. Any service enabler can access this information. Concrete examples are the Multimodal Delivery Control System that is responsible for rendering content on nearby devices and the DCS Viewer that provides a graphical user interface for accessing nearby resources.

\section{Summary}

In this paper we have presented the Mobile Ontology, an effort within the IST project SPICE to develop a standardized ontology for mobile service delivery in NGNs. The main goal of the Mobile Ontology is to provide enough flexibility and extensibility while at the same time trying to converge to a standardized semantic model for use in future Service Delivery Platform (SDP). Our approach is based on a minimal core ontology that defines common concept for sub-ontologies of relevant domains. 
We have motivated some sub-ontologies that are currently under development in the SPICE project and described some examples of their usage. The Mobile Ontology is still in an early phase and will need to be extended to further domains. We therefore greatly encourage other projects to use and actively contribute to this important effort so that NGN SDP providing value added service to its users can become a reality and could be standardized. An entry point for further details as well as the ontology files is available at http://ontology.ist-spice.org/.

Acknowledgments. This work has been performed in the frameworks of the IST project IST2005027617 SPICE, which is partly funded by the European Union. We would like to thank all SPICE project partners, especially people that have been involved in the ontology work. We would also like to thank to Martin Bauer for explaining us the MAGNET ontology and providing us detailed and early access to it.

\section{References}

1. Fensel, D.: Ontologies: A Silver Bullet for Knowledge Management and Electronic Commerce. Springer, Heidelberg (2001)

2. Chen, H., Perich, F., Finin, T., Joshi, A.: SOUPA: Standard Ontology for Ubiquitous and Pervasive Applications. In: The Proceedings of the First Annual International Conference on Mobile and Ubiquitous Systems: Networking and Services (Mobiquitous 2004), Boston, MA, August 22-25 (2004)

3. Heckmann, D., Schwartz, T., Brandherm, B., Schmitz, M., von Wilamowitz-Moellendorff, M.: GUMO - the general user model ontology. In: Ardissono, L., Brna, P., Mitrović, A. (eds.) UM 2005. LNCS, vol. 3538, pp. 428-432. Springer, Heidelberg (2005)

4. Niles, I., Pease, A.: Towards a Standard Upper Ontology. In: Welty, C., Smith, B. (eds.) Proceedings of the 2nd International Conference on Formal Ontology in Information Systems (FOIS 2001), Ogunquit, Maine, October 17-19 (2001)

5. Jacobson, M., et al.: Specifications of PN networking and security components, MAGNET Beyond Public Deliverable D2.3.1, http: / / www. ist-magnet.org/public+deliverables/BeyondwP2

6. Zhdanova, A.V., et al.: Ontology Definition for the DCS and DCS Resource Description, User Rules. SPICE deliverable D3.1 (October 2006)

7. Tarkoma, S., et al.: Unified Mobile Platform for Service Mash-Ups. Accepted at Mobility Conference 2007, Singapore, September 10-12 (2007)

8. Almeida, J.P., Baravaglio, A., Belaunde, M., Falcarin, P., Kovacs, E.: Service Creation in the SPICE Service Platform. In: The Proceedings of the 17th Wireless World Research Forum Meeting (WWRF17), Heidelberg, Germany, November 15-17 (2006)

9. Martin, M., et al.: Service Enablers demonstration (mock-ups highlighting service intelligence in an outdoor extended LBS scenario). SPICE deliverable D4.4 (September 2006)

10. Strohbach, M., Goix, L.W., Kovacs, E.: Integrating IMS Presence Information in a Service Oriented Architecture. In: The Proceedings of KiVS 2007 (2007) 